\section{The Early History of Arimoclomol for Inclusion Body Myositis}

Mazen M. Dimachkie, Pedro M. Machado, Claus

Sundgreen, Thomas Blaettler, Jeffrey Statland,

Andrew Heim, Laura Herbelin, Linda Greensmith, Michael G. Hanna and Richard J. Barohn

\section{Background}

The early history of arimoclomol involves two parallel lines in exploration, one in the USA and the other in the UK. In the mid-2000s, Dr. Rick Barohn was asked to be a site in a Phase 2 study of arimoclomol in Amyotrophic Lateral Sclerosis (ALS). This was a Northeast ALS Consortium (NEALS) study that was funded by the drug company CytRx, which owned the compound. CytRx was interested in the possibility that arimoclomol could slow down the progression of neurodegenerative diseases that involved protein misfolding. ${ }^{1}$ This hypothesis was based on the 2004 findings from Dr. Linda Greensmith's lab in the UK, which showed that arimoclomol was effective at delaying disease progression and extending lifespan in the $\mathrm{SODI}^{\mathrm{G} 93 \mathrm{~A}}$ mouse model of ALS. ${ }^{2}$ Dr. Barohn attended the investigators meeting, learned about the drug, and had the idea that it should also be tried in sporadic inclusion body myositis (IBM). He discussed this with the CytRx representatives. They had not heard of IBM but were interested, and Dr. Barohn sent them information about the disease including the Muscle Study Group (MSG) publications.

\section{Targeting Protein Homeostasis in Sporadic Inclusion Body Myositis - A KU \& UK Collaboration}

In the fall of 2006, Dr. Barohn and his research assistant, Dr. Jeffrey Statland (who had just begun the internship), presented the concept of using arimoclomol for IBM at the annual MSG meeting in Beaver Hollow, N.Y. They explained the rational of using the drug for IBM. He was asking if the MSG would consider becoming involved in a multicenter trial (see Appendix). They proposed a 70 patient, one-year trial of $300 \mathrm{mg} /$ day and proposed the primary outcome would be quantitative muscle testing (maximum voluntary isometric contraction testing [MVICT]) with secondary outcomes of IBM Functional Rating Scale (IBMFRS), time to walk 15 feet, manual muscle testing (MMT), Purdue peg placement, 36-Item Short Form Survey (SF-36), and Individualized Neuromuscular Quality of Life Questionnaire (INQoL). They proposed that the known natural history of IBM is that the MVICT scores will decline by an average of $4 \%$ or $\mathrm{Z}$ score of 0.28 over 6 months. They extrapolated a Z score for 12 months of 0.6 units and stated that to halt progression they would need a sample size of 35 subjects per group to detect a difference between the 2 groups, with $80 \%$ power. They suggested an optional muscle biopsy sub-group to examine Heat Shock Protein 70 (HSP70) levels, inflammation, vacuoles, and amyloid inclusions. Unfortunately, the feedback from the MSG was disappointing. There was little enthusiasm for the project moving forward at that time as a multicenter trial. It was suggested a single site trial should be the initial step.

Dr. Barohn then applied to CytRx for an investigatorinitiated grant so that the company would supply the University of Kansas Medical Center (KUMC) team with enough drug and placebo to study 12 patients with IBM in a pilot trial to study tolerability in this patient population. Dr. Barohn created the protocol with several investigators at KUMC including Dr. Jeff Statland, Dr. Yunxia Wang, and Laura Herbelin. Dr. Wang obtained internal pilot funding from the General Clinical Research Center (GCRC) National Institutes of Health (NIH) pilot grant program for junior investigators to perform the study on the GCRC at KUMC. Dr. Barohn applied to the Food and Drug Administration (FDA) to get an Investigational New Drug (IND) designation (FDA-IND \# 76,773) and he was able to cross-file on the CytRx IND. He asked the FDA if a 12-month trial could be accomplished. The FDA at the time only had human data from ALS patients receiving the drug for 3 months, so they would not give him the 12-month study. The FDA would only agree to a 4-month drug study. Therefore, a protocol was designed whereby the patient received drug or placebo for 4 months, but they were followed on the protocol for another 8 months, or a total of a year. Patients had monthly evaluations. In addition to looking for safety signals they also proposed to do preand post-muscle biopsies to measure HSP70 levels and histologic features as noted above. The biopsies were to be obtained at baseline and after Month 4 .

A parallel interest in the drug for IBM was occurring in the UK. The only individual who was enthusiastic about the Barohn and Statland presentation at the MSG in 2006 was Dr. Michael Hanna, who had heard about the drug from his colleague Dr. Greensmith. She had been working with arimoclomol and its derivatives for several years in collaboration with Biorex, the original Hungarian company that made arimoclomol, testing its neuroprotective properties in a number of mouse modes of motor neuron degeneration, including the $\mathrm{SODl}^{\mathrm{G} 93 \mathrm{~A}}$ mouse of ALS. It was these findings in $\mathrm{SODl}^{\mathrm{G} 93 \mathrm{~A}}$ mice that led CytRx to acquire arimoclomol and set up the first trials of the drug in ALS patients. Dr. Greensmith's findings in ALS led her also to test the effect of arimoclomol in models of other protein misfolding disorders, including models of IBM. Her findings showed that arimoclomol was able to decrease levels 
of abnormally folded proteins and prevent cytoplasmic mislocalisation of TDP-43 in primary rat muscle cell cultures transfected with beta amyloid precursor protein or exposed to inflammatory mediators. Furthermore, arimoclomol was also found to ameliorate the disease phenotype of mice expressing mutant Valosin Containing Protein (VCP), in which skeletal muscles show an inclusion body myopathy-like phenotype, including accumulation of misfolded proteins as well as muscle atrophy and weakness. Dr. Hanna approached CytRx to obtain some arimoclomol for a small clinical trial at the UCL Queen Square Institute of Neurology. Dr. Barohn, however, had already approached the company and had an agreement with them to receive the drug and do a pilot trial in Kansas. Since Drs. Barohn and Hanna were both leaders in the MSG, they decided to join forces and do a 24 patient IBM study, 12 in London and 12 in Kansas City with a 2-to-1 randomization of active drug to placebo. CytRx was willing to expand the size of the study if it was going through one protocol and one IND, held by Dr. Barohn.

Dr. Hanna found national funding from Arthritis Research UK (merged in 2018 with Arthritis Care to form Versus Arthritis) to perform the study in London at the UCL Queen Square Institute of Neurology. In 2007, Drs. Barohn and Statland obtained an Rl3 NIH grant to have an international meeting on nondystrophic myotonias in Kansas City. Dr. Hanna's group was also part of that project and they attended the meeting. This allowed both doctors to finalize the protocol so that each site was enrolling patients by the fall of 2007 on both continents. Dr. Dimachkie also met with Dr. Barohn and Dr. Hanna; he was introduced to the study as he was moving to Kansas City to start his new position at the KUMC. Once Dr. Dimachkie formally joined Dr. Barohn at KUMC that summer, he became involved in the study. Dr. Adrian Miller and subsequently Dr. Pedro Machado became involved in the study at Queen Square assisting Dr. Hanna.

Meanwhile, Dr. Greensmith continued to obtain promising data in her laboratory using arimoclomol in the VCP model and in cell culture. Patients were entered into the study and completed the study over two years. The data from both sites was analyzed in London and it was determined the drug was well tolerated in this population. It was also shown there may have been a small trend at 8 months in some of the clinical endpoint measures, namely the IBMFRS, ${ }^{3}$ mean right grip strength hand grip MVICT score, and the mean MMT score. Unfortunately, the muscle biopsy measures did not show the anticipated change over time, but there were a host of reasons for that including transportation issues of the muscle tissue from the USA to UK, which may have accounted for this. After extensive discussion, it was decided to merge the clinical data and the pre-clinical data into one manuscript. This manuscript was published in Science Translational Medicine in 2016. ${ }^{4}$

\section{The IBM4809 Path Forward - A Randomized Controlled International Efficacy Study}

As the group decided to publish the combined results of the preliminary data, the group moved to apply for a federal grant to fund a larger multi-center international randomized controlled study. When the data was presented at the MSG there was growing enthusiasm to move forward. A protocol was designed using the IBMFRS as the primary endpoint instead of MVCIT for the large trial, and the sample size was increased to 150 subjects in a 1-to-1 randomization. The protocol was approved by the MSG executive committee and it became an official MSG study. The project was presented to the NIH NeuroNext consortium and was rejected for a full grant application. Dr. Dimachkie had become the overall PI of the project by this time with Dr. Machado as the multiple PI and Drs. Hanna and Barohn in senior leadership roles. Dr. Dimachkie then applied to the FDA Orphan Products Development (OOPD) program for funding. The application was funded on the third try, and Dr. Dimachkie received notice of the grant award in 2015 (RO1FD004809), which we named IBM4809. The trial is registered as NCTO2753530. ${ }^{5}$

In the meantime, CytRx sold the rights to the drug to the Danish company Orphazyme. The 4 trial leaders began meeting regularly with Orphazyme to explain the nature of the IBM project and to get assurance that they could get both drug and placebo for the project. This relationship developed well, and the company was interested in the preliminary data from the pilot study. Orphazyme performed a post hoc responder analysis of the efficacy data from the pilot study (Figure) based on the yearly average rate of decline of the IBMFRS by 3 points. This demonstrated that $14 / 16$ in the arimoclomol $100 \mathrm{mg}$ PO T.I.D. group did not progress $(n=9)$ or improve $(n=5)$ at 8 months, which was 4 months after the last randomized dose. However, $2 / 16$ progressed as assessed by a loss of 2 or more points on the IBMFRS at 8 months. In the placebo group, $2 / 8$ were stable (1) or improved (1) at 8 months; $6 / 8$ progressed. This encouraging post hoc analysis furthered the already existing strong enthusiasm of this collaborative group.

Ultimately, and as detailed later in this report, Orphazyme became interested to the point that the company suggested this become a joint industry-academia study moving forward, and the investigators agreed. Dr. Barohn transferred the IND to Orphazyme. Orphazyme and Dr. Dimachkie had interactions with the FDA regulatory agency. One outcome from these meetings is that the FDA 
Figure 1. Post hoc analysis of pilot study data.

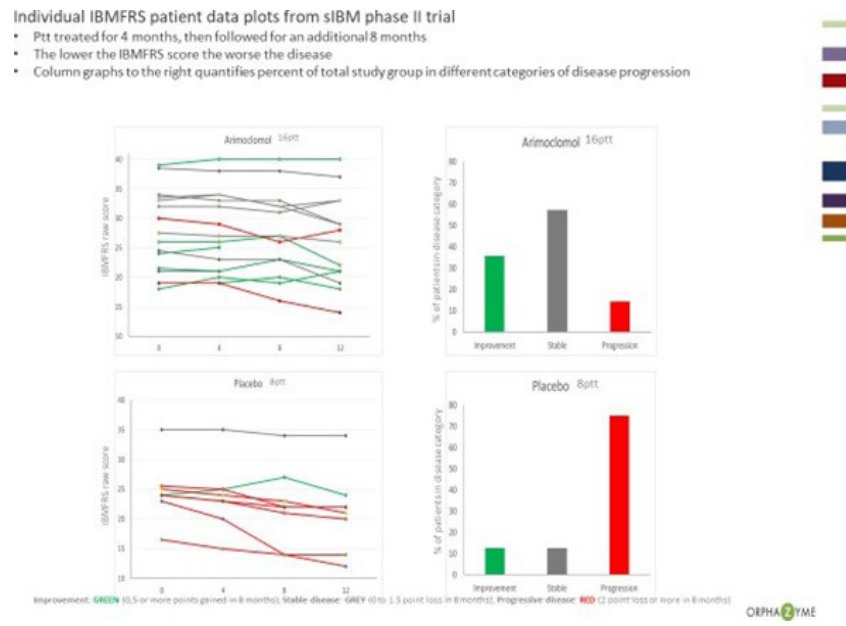

accepted the IBMFRS as a clinically relevant primary outcome measure for this study.

Despite receiving FDA-OOPD notice of award to start June 1, 2015, Dr. Dimachkie did not initiate enrollment as several important next steps needed to be addressed. Given the limits on maximum FDA-OOPD funding dollar amounts at that time, this study of arimoclomol in IBM did not yet contain the "bells and whistles" to fulfill a strict regulatory agency's approval pathway. If this study were to be positive, it would be very important to have a more rigorous structure such as Code of Federal Regulations (CFR) Part 11 compliant database or on-site monitoring. On July 17, 2015, Dr. Dimachkie met with the Orphazyme leadership team in Boston and was joined via teleconference by Dr. Barohn and Dr. Hanna to share the good news. Dr. Dimachkie presented the study data and shared news about the FDA-OOPD grant award. As part of the grant application to FDA-OOPD, Orphazyme had already made a commitment to manufacture matching capsules of arimoclomol and placebo to be released to the trial through several campaigns. However, the goal of the Boston meeting was also to discuss with Orphazyme the possibility of expanding the scope of this academia-industry collaboration.

Intense communication with Orphazyme followed this meeting through the rest of 2015 . This culminated in the collaboration of the study team with Orphazyme to support the Type $\mathrm{C}$ filing with the FDA regulatory division. On March 18, 2016, Dr. Dimachkie and Laura Herbelin met in New Jersey with Orphazyme and B\&H Consulting Services, Inc. The Type C file was finalized shortly thereafter and submitted by B\&H, requesting a meeting with US Regulators for the middle of 2016. Later that year, the collaborative group exchanged correspondence with the FDA regulatory division and adjusted the protocol according to their feedback. As an example, we were encouraged to seek a higher dosage of arimoclomol. We evaluated the current safety data of arimocolomol. Based on that analysis, we informed the FDA regulatory agency that we were increasing the dosage to $400 \mathrm{mg}$ PO TID. The protocol was adjusted to match requirements of section 6 of the International Conference on Harmonisation-Good Clinical Practice (ICH-GCP) rules. All along the way, Dr. Dimachkie was in close communication with the FDAOOPD program manager to keep the FDA-OOPD funding agency informed of ongoing work and to make sure that any proposed changes do not compromise existing study funding. By late 2016, Orphazyme provided more study support by funding a CFR Part 11 compliant database and hiring a Clinical Research Organization (CRO) to perform on-site monitoring.

The next challenge was to get the drug delivered from Europe to the US. The study drug was manufactured in France and packaged in Germany for shipment. To that point, Dr. Dimachkie got the support of the KUMC Research Institute, Inc (RI). To become the Importer of Record and after seeking guidance from experienced importers, Dr. Dimachkie and the KUMC RI submitted the application to allow investigational medical product entry into the US in order to enroll the first study subject. In the summer of 2017, the study drug shipment cleared customs and arrived in Kansas. KUMC remained the Importer of Record until Orphazyme took over IND sponsorship in late 2017 and managed drug entry into the US and UK for the remainder of the study. By mid to late 2018, all participating sites were activated. To further support the 2014 orphan designation by the FDA OOPD, Orphazyme received in December 2019 FDA Fast Track designation for arimoclomol in IBM. Orphan designation (EU/3/16/1659) had also been granted by the European Commission/European Medicines Agency (EMA) on May 30, 2016, following an application and physical meeting with the EMA on April 19, attended by Dr. Pedro Machado, Dr. Linda Greensmith, and Orphazyme representatives.

\section{Conclusions}

In summary, this strong academia-industry collaborative partnership with Orphazyme is fundamental not only for providingan experimental drug, butalsosecuring robust operational trial conduct and GCP compliance. This strong partnership drove regulatory interactions and processes and allowed for the implementation of add-on studies to investigate pharmacokinetics, introduce clinician and patient global impression scales, a muscle MRI substudy, and most importantly, an open-label extension opportunity for the 4809 participants. All along the way, 
the ultimate goal has been to move forward the field of IBM with strong communication that promotes lasting and trusting partnership. If the IBM4809 study results are positive, this strong partnership is positioned to support filing for regulatory approval in the US and Europe, with the possibility to offer the first effective therapy for people with IBM.

\section{Corresponding Author}

Mazen M. Dimachkie, M.D. mdimachkie@kumc.edu

\section{Acknowledgments}

We acknowledge funding support from the Neurology Department Ziegler Research Fund and the NIH Funded General Clinical Research Center for the pilot study conduct at the KUMC. We also recognize funding from the FDA-OOPD (RO1FD004809) and support for Ophazyme for the IBM4809. PMM and MGH are supported by the National Institute for Health Research (NIHR), University College London Hospitals (UCLH), Biomedical Research Centre (BRC). We thank patients with IBM who have participated in the pilot study and in IBM4809 and all of those who have expressed interest in the studies.

\section{References}

1. Cudkowicz ME, Shefner JM, Simpson E, Grasso D, Yu H, Zhang H, Shui A, Schoenfeld D, Brown RH, Wieland S, Barber JR; Northeast ALS Consortium. Arimoclomol at dosages up to $300 \mathrm{mg}$ /day is well tolerated and safe in amyotrophic lateral sclerosis. Muscle Nerve. 2008;38(1):83744. doi: 10.1002/mus.21059. PMID: 18551622.

2. Kieran D, Kalmar B, Dick JR, Riddoch-Contreras J, Burnstock G, Greensmith L. Treatment with arimoclomol, a coinducer of heat shock proteins, delays disease progression in ALS mice. Nat Med. 2004;10(4):402-5. doi: 10.1038/nml021. Epub 2004 Mar 21. PMID: 15034571.

3. Ahmed M, Machado PM, Miller A, Spicer C, Herbelin L, He J, Noel J, Wang Y, McVey AL, Pasnoor M, Gallagher P, Statland J, Lu CH, Kalmar B, Brady S, Sethi H, Samandouras G, Parton M, Holton JL, Weston A, Collinson L, Taylor JP, Schiavo G, Hanna MG, Barohn RJ, Dimachkie MM, Greensmith L. Targeting protein homeostasis in sporadic inclusion body myositis. Sci Transl Med. 2016;8(331):331ra4l. doi: 10.1126/scitranslmed.aad4583. PMID: 27009270 ; PMCID: PMC5043094.

4. Jackson CE, Barohn RJ, Gronseth G, Pandya S, Herbelin L; Muscle Study Group. Inclusion body myositis functional rating scale: a reliable and valid measure of disease severity. Muscle Nerve. 2008;37(4):473-6. doi: 10.1002/mus.20958. PMID: 18236463.

5. Study of Arimoclomol in Inclusion Body Myositis. Trial details publicly available at: https://clinicaltrials.gov/ ct2/show/NCT02753530 


\section{Appendix}

PowerPoint slides presented by Drs. Jeffrey Statland and Richard Barohn at the 2006 Muscle Study Group meeting.

\section{Arimoclomol Therapy for IBM}

Richard J Barohn, MD

Jeffrey Statland, MD

Department of Neurology

University of Kansas Medical Center

\section{Arimoclomol Therapy for IBM}

- IBM was thought to be an immune mediated myopathy

- IBM does not respond to Immuno-mudulatory therapy

- IBM is increasingly thought to be a disease of myodegeneration

- Protein misfolding may play a role in the pathology of IBM

- Arimoclomol is a potent co-inducer of Heat Shock Proteins

- Heat Shock Proteins protect the cell from protein misfolding

\section{Primary Outcome Measure}

- A 12-month placebo controlled double blind trial of Arimoclomol 300mg/day versus placebo in 70 patients with IBM

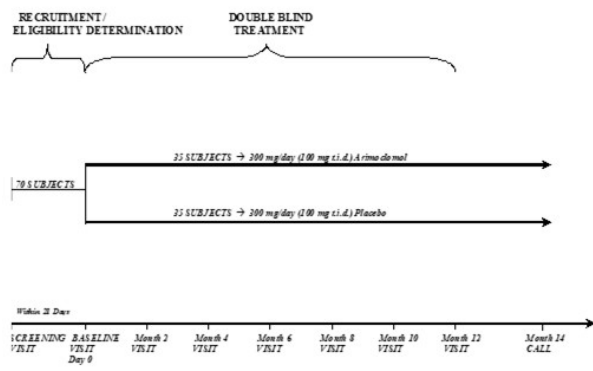

\section{Secondary Outcome Measures}

- Manual Muscle Testing

- Purdue Peg Placement

- Time to walk 15 feet

- IBM-FRS

- SF-36 v2

- Functional Disability Scale

- Will add INQoL to protocol

\section{Statistical Considerations}

- Natural history and Avonex studies suggest MVICT scores will decline by an average of $4 \%$, or $0.28 \mathrm{Z}$ score units over 6 months (SD 0.88)

- Extrapolating to 12 months, we expect a decrease of $0.6 \mathrm{Z}$ score units

- To halt progression need sample size of 35 subjects per group

- This will provide $80 \%$ power to detect a difference in the mean response of $0.6 \mathrm{Z}$ score units between the two groups, using a two-tailed two-sample ttest at the 0.05 level of significance 


\section{Muscle Biopsy Sub-Study}

- Optional study in 35 volunteers

- Month 12 versus Baseline, drug versus placebo

-Will examine:

$\square$ HSP 70 levels (Gallagher)

$\square$ Inflammation

$\square$ Vacuoles

$\square$ Amyloid inclusions

$\square$ Gene expression profiles (Greenberg)

\section{Background and Preliminary Studies}

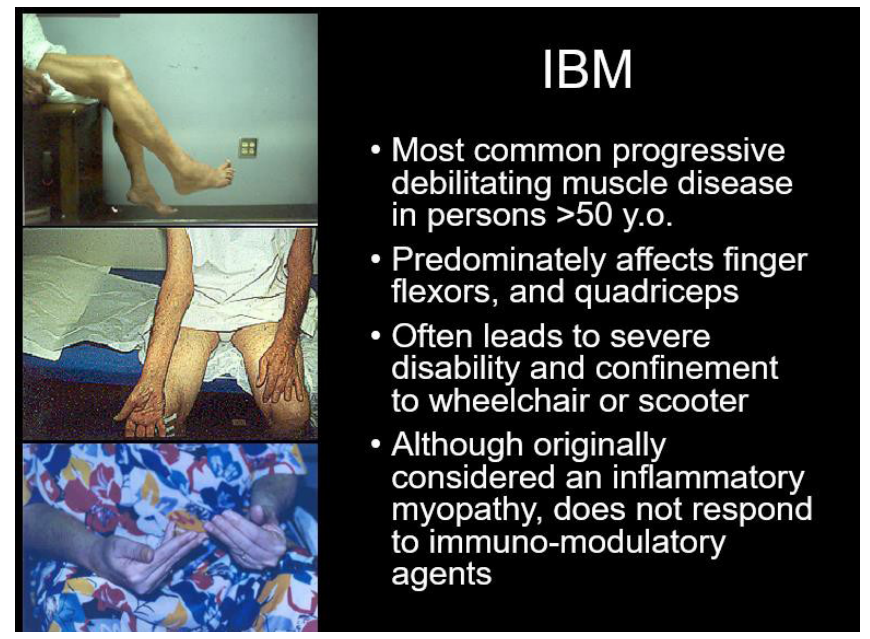

IBM may be a Myodegenerative Disorder

- Muscle Biopsies show:

- Amyloid fibrils containing A $40 / 42$

- Paired helical filaments containing p-tau

- These inclusions co-localize with other proteins also found in Alzheimer's disease

- Presenelin-1

- Apo E

- Ubiquitin, etc

- In vitro models overexpressing A $\beta$ Precursor Protein mimic IBM pathology

- Askansas. Neuroreport. 1997; 8: 2155-2158.

- Animal models overexpressing A $\beta$ Precursor Protein mimic IBM pathology

- Fukuchi. Am J Pathol. 1998; 153: 1687-1693.

- Jin. A, J Pathol. 1998; 153: 1679-1686

\section{IBM Pathology}

- Inflammatory cells

- Vacuoles

- Amyloid inclusions

- Paired helical

filaments
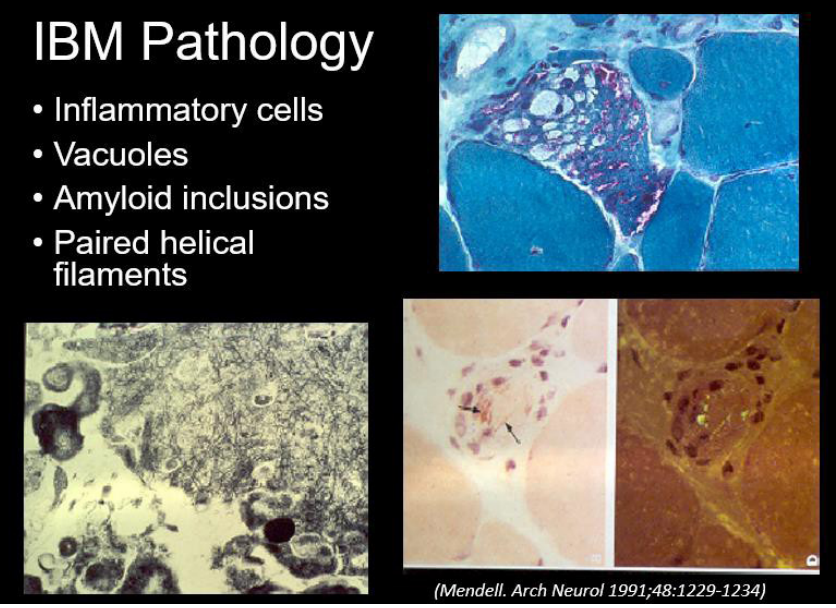

\section{Arimoclomol}

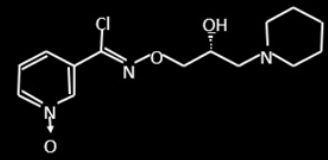

- Derivative of Bimoclomol, developed by CytRx, a potent co-inducer of Heat Shock Proteins

- Stabilizes Heat Shock Transcription Factor-1 (HSF-1) - This increases levels of HSP70 and HSP90

- Interacts with acidic membrane lipids to stabilize plasma membranes

- Interacts with cardiolipin in mitochondria

- May stabilize membrane

- May inhibit apoptosis

(Kieran. Nature Med. 2004; 10: 402-405; Torok. PNAS. 2003; 100: 3131-3136) 


\section{Heat Shock Proteins}

- Prevent aggregation of mutant and damaged proteins

- Catalyze protein folding and multimer assembly

- Solubilize aggregated proteins

- Promote ubiquitination and degradation of abnormal proteins

- Promote proper folding and glycosylation of membrane and secreted proteins

- Suppress apoptotic program

- Regulate own expression in cytosol and ER

(Sherman. Neuron. 2001; 29: 15-32)

\section{Heat Shock Pathway}

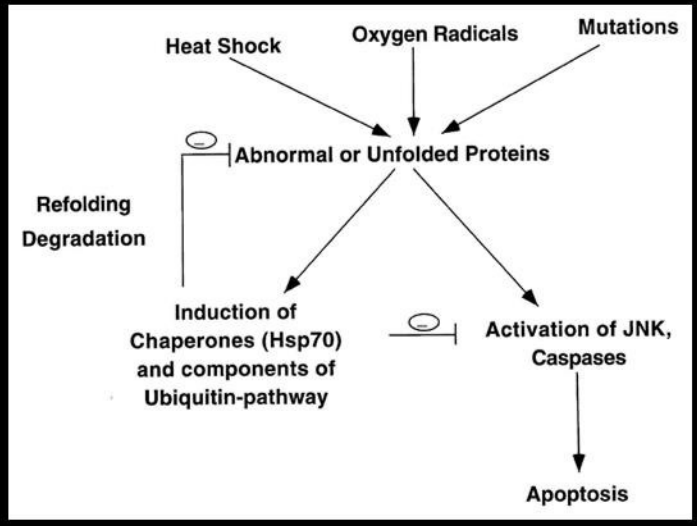

(Sherman. Neuron. 2001; 29: 15-32)

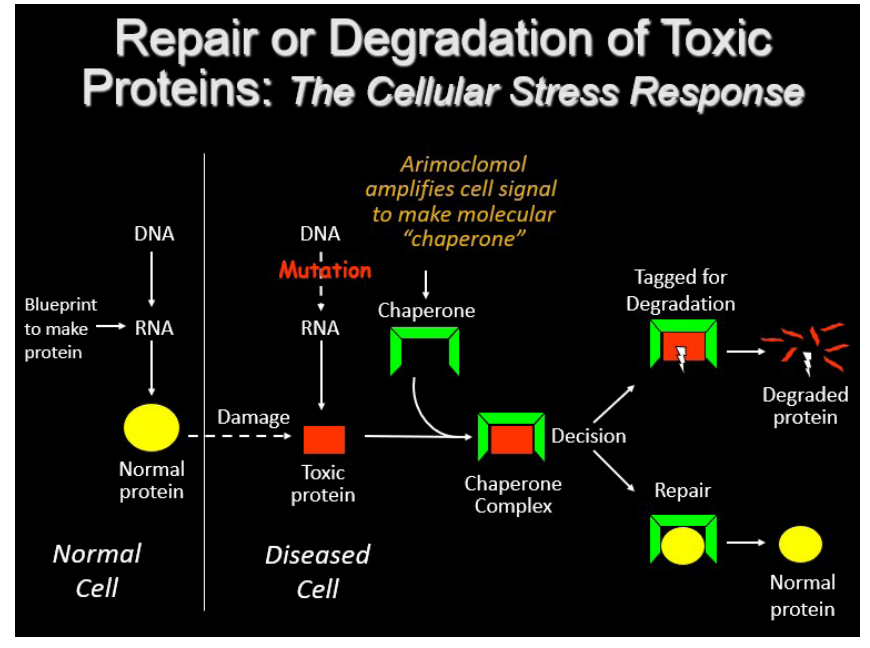

Arimoclomol Increases Lifespan in SOD1 Mice
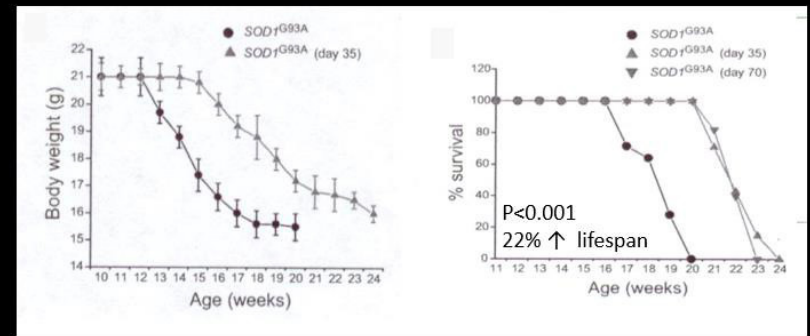

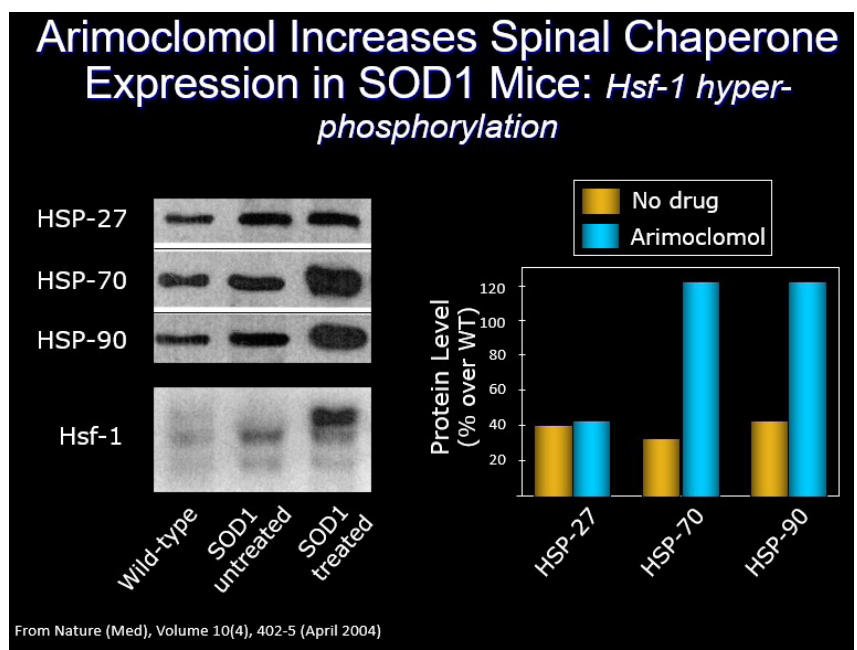

\section{Arimoclomol: Drug Characteristics}

- Chemically stable

- Bulk API stable for at least 4 years, light sensitive

- Stable in solution for at least 1 week at pH 1-13

- 2-year stability as capsules $(50,100,200 \mathrm{mg})$

- Extensive preclinical evaluation

- Safety tested in multiple species

- Good oral bioavailability, distribution, and metabolism

- Shown to be well-tolerated in two Phase I

clinical trials

- 30 total volunteers

- Well absorbed, distributed and cleared

- Currently in Phase II trial in ALS 


\section{Why Propose Arimoclomol for IBM?}

- HSP70 co-localizes with amyloid inclusions in IBM biopsies

- HSP70 levels are increased $(\sim 4.5 \mathrm{X})$ in IBM biopsies

- Protein misfolding is considered part of the pathological mechanism

- Mitochondrial defects are associated with IBM

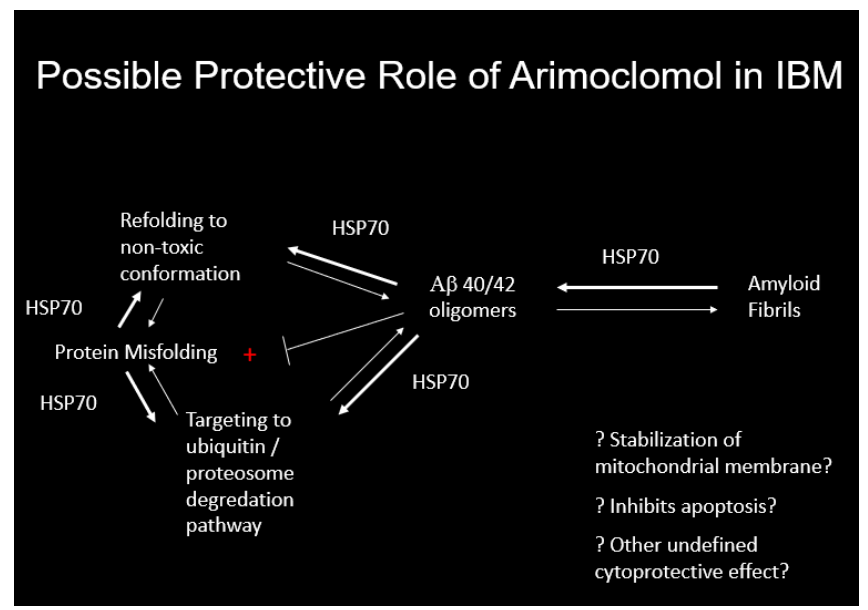

The Molecular Chaperone $\alpha$ B-crystallin Enhances Amyloid $\beta$ Neurotoxicity

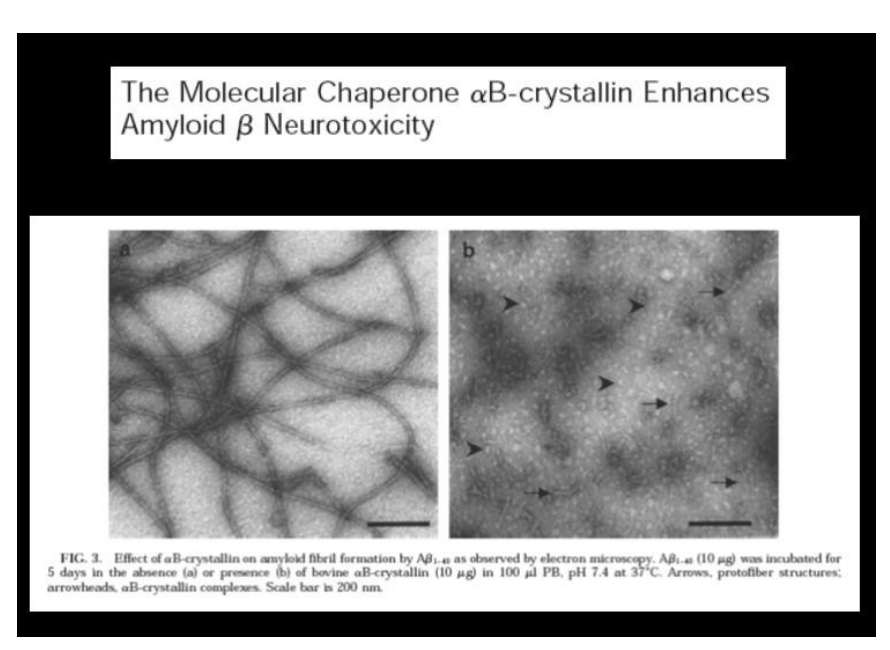

Proposed Pathological Mechanism for IBM

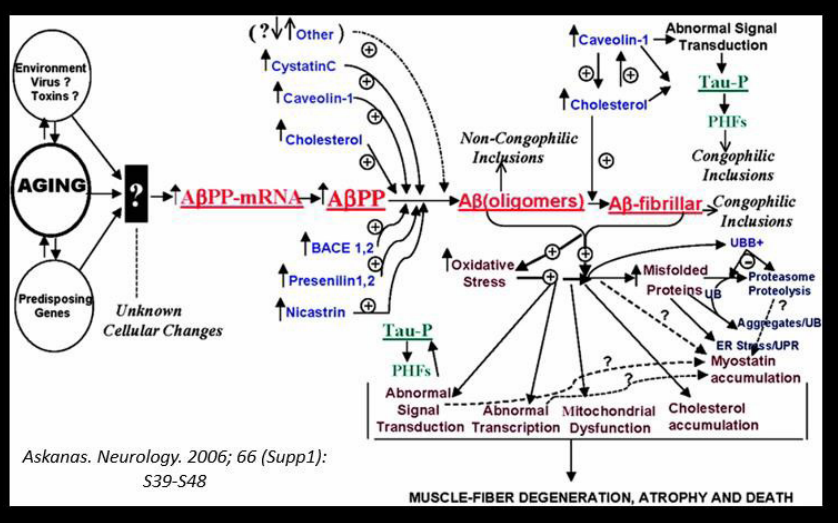

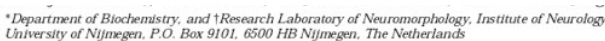

Abstract

Amyloid $\beta(A \beta)$ is a 40 - to 42 -residue peptide that is implicated in the pathogenesis of Alzheimer's Disease (AD). As a result of conformational changes, $A \beta$ assembles into neurotoxic fibrils deposited as 'plaques' in the diseased brain. In $A D$ brains, the small heat shock proteins (sHsps) $\alpha \mathrm{B}$-crystallin and $\mathrm{Hsp} 27$ occur at increased levels and colocalize with these plaques. In vitro, sHsps act as molecular chaperones that recognize unfolding peptides and prevent their aggregation. The presence of sHsps in $\mathrm{AD}$ brains may thus reflect an attempt to prevent amyloid fibril formation and toxicity. Here we report that $\alpha \mathrm{B}$-crystallin does indeed prevent in vitro fibril formation of $A \beta 1-40$. However, rather than protecting cultured neurons against $A \beta 1-40$ toxicity, $\alpha B$-crystallin increases the toxic effect. This indicates that the interaction of $\alpha \mathrm{B}$-crystallin with conformationally altering A $\mathrm{B1}-40$ may keep the

latter in a nonfibrillar, yet highly toxic form.

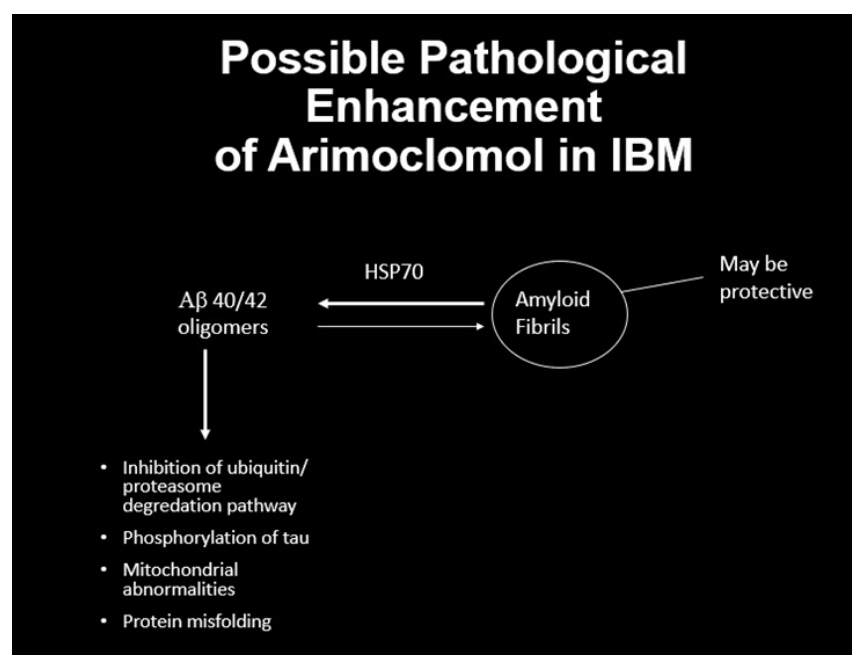




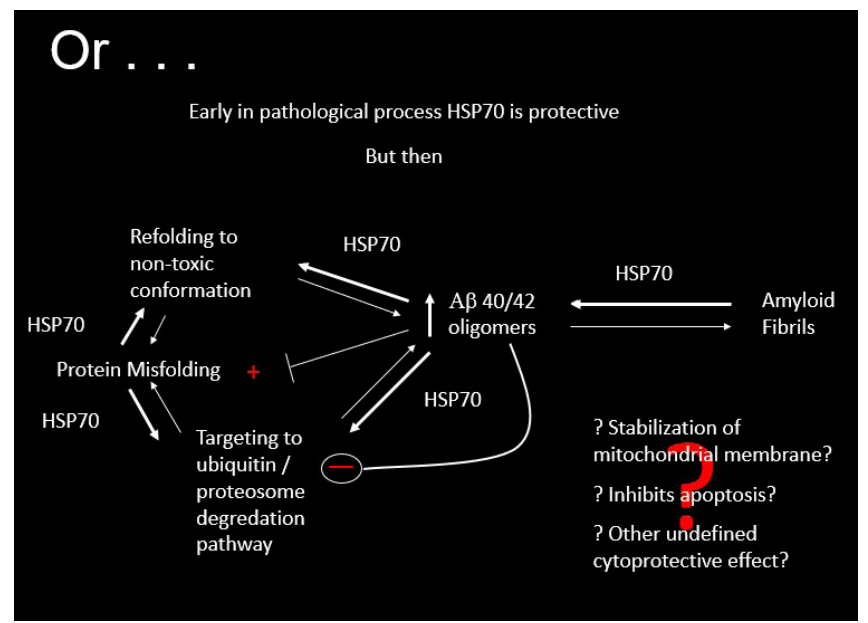

\section{Future Directions}

- Design and submit protocol grant to the NIH for an efficacy/safety/tolerability study

- May need animal or in vitro data to support grant

- NIH translational cooperation grant

- Get funding for in vitro and animal studies

\section{Implications for Arimoclomol in IBM}

- Arimoclomol may be cytoprotective

- Arimoclomol may be cytotoxic

- For muscle fibers early in the pathological process Arimoclomol may be protective

- For muscle fibers late in the pathological process Arimoclomol may accelerate cell death

\section{Leading to ...}

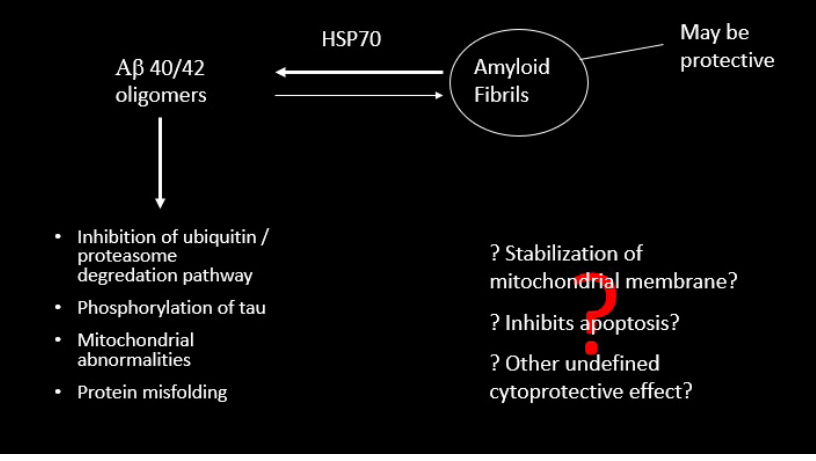

